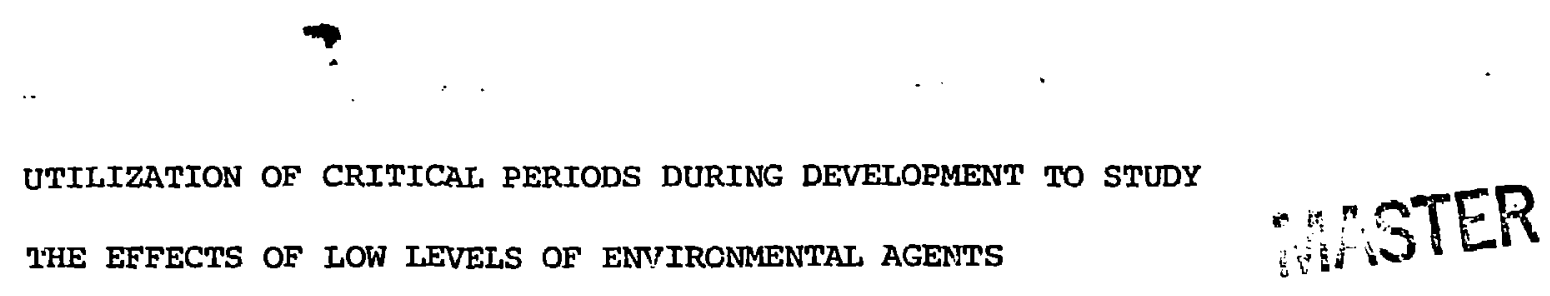

Liane B. Russell

Biology Division

Oak Ridge National Laboratory

Oak Ridge, TN 37830

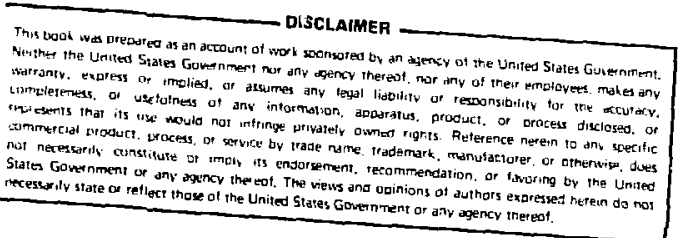

This book wa prepured as an actount of work soonsoted by a d

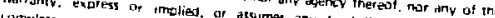
Orility tor the ration

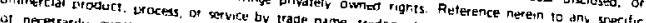

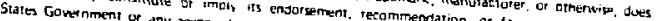
We

\title{
I. INIRODUCTION
}

The identification of susceptible subpopulations serves basic as well as applied purposes. The biological properties by which a subpopulation differs from less susceptible individuals furnish important clues about the basic mechanisms by which an environmental agent impinges on biological material of alZ organisms (e.g., repair deficiencies shed light on repair in general); they also provide information on the pathways between the original interaction and the finally expressed endpoint. From a more applied point of view, the existence of sisceptible subpopulations may lead to limitations in permissible doses of an environmental agent. Where a subpopulation cannot be physically separated from the main population, such limitations must be to the overall population. Alternatively, the susceptible subpopulation may have to be restricted from certain environments, e.g., the workplace -- a procedure that can have social and legal implications.

The developing embryo and fetus have long been known to be particularly vulnerable to ionizing radiation. In part, this excessive vulnerability is the result of the fact that a developing organism is capable of endpoints which no longer exist in an aduit. For example, one would not expect a fully formed structure (a hand,

\footnotetext{
*Research sponsored by the Office of Health and Environmental Research, U.S. Department of Energy under contract $\mathrm{w}-7405-e n g-26$ with the Union Carbide Corporation.
}

BISTRIBUTIOH OF FHIS DOCUMEKT IS UHUMITER 
or an eye) to become drastically maiformed by irradiation, whereas such an effect is quite logical when the formative stages of a structure are exposed. But even where endpoints are more directly comparable (e.g., death of the whole organism), the intrauterine inammal responds at lower doses than does the adult.

To consider the embryo as a whole as a single subpopulation is, however, a simplification that can lead to great loss in sensitivity in the detection and measurement of risk, i.e. to an underestimation of risk. Conversely, it may lead to overestimation of risk in the process of extrapolating from single to protracted exposures. The embryo is, of course, a dynamic system with rapidly changing patterns of sensitivity. As we showed 30 years ago (9, 10, 17), a given effect can be induced readizy by exposure at a well-defined developinental stage, but not at all induced by the same exposure at other stages -- even those occurring only a day before or after the sensitive one. This pattern, which we worked out for certain endpoints in the mouse, was named the pattern of critical periods.

The critical periods define subpopulations of a subpopulation whose special properties of sensitivity should be exploitable in studying risk at low levels of expcsure to environmental agents, and for the development of methods rur extrapolation. Only a limited amount of such exploitation isas occurred to date. This paper will describe three systems tinat nay be well suited for more extensive future work in this area.

II. SYSTEMS THAT UTILIZE CRITICAL PERIODS TO STUDY LOW-LEVEL EFFECTS

A. Cell Kinetics as an indicator of nervous-system maldevelopment

In man, nervous-system formation occupies a relatively much greater proportion of the period of intrauterine development than in experimental mammals, and abnormalities involving the nervous system (e.g. microcephaly, mental retardation) are thus, understandably, among the most frequently reported human teratogenic effects (16). The developing nervous system of experimental mammals has long been known to be highly sensitive (4). In spite of this, most observations have been of a qualitative nature, and little effort was made until recently to develop sensitive guantitative indicators of developmental damage.

The device of working at the stage of maximum sensitivity has led to an experimental system whose endpoints permit extrapolation to lov levels. Using at first moderate to high doses of x-rays, Kameyama, Hoshine, and Hayashi (5) investigated the undifferentiated matrix cells in the ventricle walls of the developing telencephalon 
during embryonic stages spanning a major part of cerebral-cortex formation in the mouse. They found day-13 postconception to be most vulnerable with respect to a number of parameters: (a) cellcycle changes in the first postirradiation cell division, (b) incidence of pyknotic cells $4-5 \mathrm{~h}$ postirradiation; and (c) reduction in cortical cells 7 weeks later.

Having discovered the stage of maximum sensitivity, they extended their investigations to lower doses, down to $10 \mathrm{R}$. They found that the prolongations of the $\left(\mathrm{G}_{2}\right.$ to $\left.1 / 2 \mathrm{M}\right)$ phase of the cell cycle were a linear function of the logarithm of the dose. The line did not extrapolate to zero dose, but to a point between 5 and $10 \mathrm{R}$, indicating a probable threshold below $10 \mathrm{R}$.

Although the fate of the matrix cells exhibiting an alteration of their cell-cycle times has not been directly determined, there is enough indirect evidence available to link this endpoint with damage to the cerebral cortex. Inasmuch as the finally perceived damages (e.g., cortical cell reduction in the adult, behavioral changes) are probably not as easily amenable to quantitative studies in the low dose range, the cell-cycle changes in the telencephalon, induced at the stage of maximum sensitivity, may be regarded as useful sensitive indicators of teratogenic activity.

\section{B. Docyte depletion}

The second example, like the first, deals with an effect measured at the cellular level. It illustrates, in addition, that, in order to estimate risk for the intrauterine period of man's life, it may occasionally be necessary to investigate postnatal stages in those experimental mammals that are born at a considerably less mature stage of development than is the human infant.

Our fertility studies with female mice of various ages showed that newborns did not become sterilized by doses of $x$ rays that, in young adults, caused permanent sterility after only one or two litters; but that, at early postnatal ages, the ovary appeared to be exquisitely sensitive (18). The pattern is similar when the end point is oocyte count, rather than fertility. Peters (8) found heavy destruction of mouse oocytes after only 20 R given at 7,14 , or 21 d postnatally. By administering single doses of $18 \mathrm{rad} r$ irradiation at various ages between birth and $47 \mathrm{~d}$, Doioson et al. (3) later found a distinct, and very low, minimum in oocyte survival following exposure in the second or third postnatal weeks.

Once investigators had identified the sensitive period, they were able to demonstrate effects of very low doses. In pioneering work, Oakberg (7) showed that a single dose of 3 rads $\gamma$ on day-10 postnatally significantly reduced oocyte numbers. Dose-response 
curves give no evidence for a threshold (2). Throughout the sensitive period, Lل 50 's for oocyte killing are considerably lower than any known LD50's for other types of cell death. Reported values include 8.4 rads $(2.9 \mathrm{rad} / \mathrm{min} \gamma$ irradiation) for stage-1 oocytes at 10 days (7), 7 rads ( $1 \mathrm{rad} / \min _{1} \gamma$ ) at 18 days (2), and $4.5 \mathrm{rads}$ (tritium in body water) administered through the drinking water during the entire period (2).

It should be noted that the mechanism by which oocytes are depleted is unknown. Since the cells are nondividing, depletion is not through aneuploidy death. Most oocytes of late fetal and newborn mice are in meiotic prophase (pachytene or diplotene). Shortly after birth, the cells enter the arrested, dictyate, stage, and it is this newly attained dictyate that appears to be particularily sensitive. Working at the height of the sensitive period, days 10-12, Oakberg (7) found a lower oocyte survival when irradiation was administered at $2.9 \mathrm{r} / \mathrm{min}$ than when it was given at $0.01 \mathrm{r} / \mathrm{min}$. This indicated the existence of repair processes in the newly attained dictyate oocyte. Subsequent investigations (2) clearly confirmed the dose-rate effect during the second and third prenatal weeks, but also showed that the oocyte's ability to recover was limited. The age pattern in sensitivity that has been demonstrated with radiation, appears to be closely paralleled when newborn, juvenile, or young adult mice are treated with 3methylcholanthrene (3) and appears thus to be the result of intrinsic features of the early dictyate oocyte.

While early dictyate oocytes are present during juvenile stages of the mouse, they are found during fetal stages in primates, including man (Oakberg, private communication). Oocytes of women and adult monkeys, in contrast to those of rodents, have been notoriously radioresistant; however, there is increasing evidence (summarized in ref. 3) for elevated radiosensitivity during the last trimester of primate development. Using the spider monkey, Dobson et al. (3) found an $\mathrm{LD}_{50}$ of, at most, 5.6 rads from tritiated drinking water (less, should only a portion of the last trimester be sensitive). It appears, therefore, that the sensitive test system developed for the juvenile mouse may be directly applicable to human risks.

The susceptible stages of the ovary obviously provide good experimental material for measuring risk in a subpopulation. Not only is the endpoint of clear practical importance (in that it concerns human fertility), but it is not far down the chain of events between initial lesion and scorable effect and lends itself readily to quantitative analysis. The time span during which oocyte killing can be profitably used to investigate low-level effects is a fairly long one -- perhaps 2 weeks in the mouse. This makes the end point amenable to studies involving dose fractionation or protraction. It also theoretically provides a good chance for 
detecting effects in epidemiological studies. From a practical standpoint, however, such detection is unlikely, since oocyte counting is ordinarily not feasible, and fertility impacts would probably not occur until near the end of the normal reproductive period, when few women conceive in any case.

\section{Homeotic shifts in the skeleton}

Morphological aberrations ("birth defects") are, for obvious reasons, perceived by man as major risks from prenatal exposure to harmful agents. Because of this, it seems advisable to design one or more test systems around morphological endpoints. In cloing so, one must bear in mind the possible complexity of the pathways between the initial lesion and the observed effect (Fig. 7 in ref. 17). Because of the likelihood, moreover, that several targets must be hit before a malformation pathway is even initiated (e.g., death of a single cell presumably does not produce polydactyly), simple dose-effect relations are not to be expected.

The early work on critical periods already showed that certain malformations had high thresholds, i.e., they were readily induced by $300 \mathrm{R}$, but not induced at all by $200 \mathrm{R}(9,10)$. It also demonstrated the complexity of interpreting quantitative relations when both incidence and degree of an abnormality could vary with dose (9).

.We have endeavoured to find morphological endpoints that are serviceable in a low dose range in order to make use of a sensitive subpopulation for the detection of teratogenic potential of environmental agents. One prerequisite is that such endpoints should be suitable for quantitative analysis, with incidence and degree being part of the same scale.

Certain problems with, as well as opportunities for, quantitative analysis of morphological effects are illustrated in Fig. 1. It may be assumed that the genotype fixes the 1 ssition of the mean value on a scale of developmental potencies. Variability about this mean is caused by a multiplicity of normally occurring small environmental variables in development. Body weight is a readily comprehended example of such a situation, in which the finally observed character -- as well as the underlying developmental potencies -- are capable of continuous variation. Where, however, the final character must vary by discreet steps, rather than continuously, as is the case for many quantitative morphological features (e.g., 13 vs. 14 ribs, 7 vs. 8 teeth), canalization occurs through the superimposition of thresholds, and the areas uncer the curve on each side of the threshold can be translated into histograms. This type of analysis is based on Sewall wright's 
Fig.1. Hypothetical distributions relating to a specific character in three strains of mice, A, B, and C. -- The top portion of the figure illustrates continuous variability about a mean, fixed by the genotype, on a scale of developmental potencies. Solid lines represent normal position, broken lines new position following an environmental interference in development; in this example, all three strains are shifted to the same degree. - The bottom portion of the figure illustrates distribution of a discontinuously varying character, derived from the continuous distributions shown in the top portion when presence of a threshold causes canalization. The histograms are derived from the areas under the curve on each side of the threshold. Solid and broken lines, as well as strain designations, correspond to top portion of the figure. -- Although the treatnent in this hypothetical case has shifted all strains equally, strain $B$ is the most useful of the three for quantitative studies of small effects (see text). 

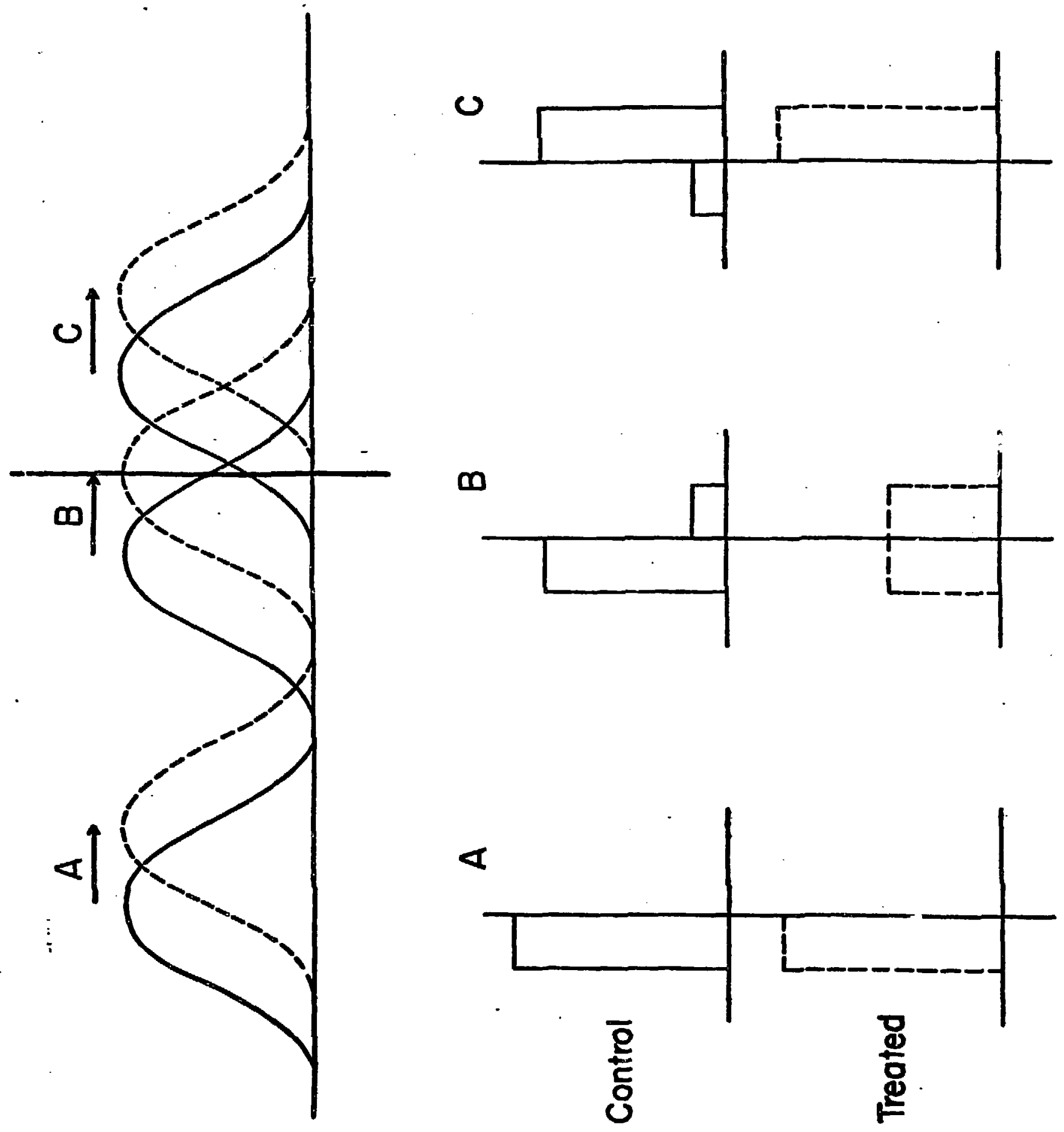
classical study of toe development in different inbred strains of guinea pigs $(19,20)$.

One may note, by looking at distributions $A, B$, and $C$, that shifts of the same magnitude can have ver $Y$ different probabilities of being detectable and/or measurable. In the case of $A$, the shift would not be detectable at all, because neither the old nor the new distribution crosses the threshold; in the case of $B$, the shift would be both detectable and measurable; while, in the case of $C$, the shift would be detectable but not measurable, since the new distribution no longer crosses the threshold, and the position of the mean can thus not be fixed. The ideal situation to aim for is thus $B_{i}$ that means finding an inbred strain that possesses a great deal of normal variability with regard to the character being studied.

During our early exploration of critical periods, we found that the development of the last rib (thoraco-lumbar border) could we affected in different ways by irradiation administered at different stages. Thus, exposure on day $8 \frac{1}{2}$ postconception (p.c.) shifted the border posteriorly, while exposure on day $11 \frac{1}{2}$ p.c. shifted it anteriorly $(10,17)$. These and similar numerical effects have been referred to as homeotic shifts (12). The strain of mice used in the early investigations (actually, an $F_{1}$ between two inbred strains) was, in the absence of radiation, quite invariable with regard to this feature and to other quantitative characteristics of the axial skeleton. The constancy indicated that this genotype probably did not provide the most favorable material for working with the desired end points (cf. strain A in Fig. 1). A subsequent exploration of other strains led us to select the BALB/C.

The question of stage sensitivity for the homeotic shifts in the axial skeleton was reinvestigated using the BALB/C, since different genotypes may have slightly different developmental timetables. As had been the case for the (C57BL $\times \mathrm{NB}) \mathrm{F}_{1}$ in our earlier work $(10,17)$, the $B A L B / C$ was quite sensitive on day 81 to the induction of posterior shifts. Clearcut effects had been found with $25 \mathrm{R}$ (11), the lowest dose then tested. The stage of maximum sensitivity, however, has recently been establisked to be 18 hours later, on day $9 \frac{1}{4}$ (12). This was true of 4 different characters: position of the thoraco-lumbar border, position of the lumbo-sacral border, number of sternebrae, and number of costo-sternal junctions (Fig. 2). (The first of these characters showed an anterior shift when treatment was three days later, day 12t.)

The BALB/C strain on day $9 \frac{1}{4}$ p.c. thus provides a potentially sensitive system for the detection of environmental insults to the embryo. We have concentrated on day $9 \frac{1}{4}$ and are in process of determining the lowest dose of $X$-rays that can be readily detected. For two of the four characters, clearcut shifts are apparent after 
Fig.2. Frequencies of various quantitative skeletal characters in newborn BALB/C mice following $100 \mathrm{R}$ irradiation at different stages in embryonic development. (Reproduced, by permission, from ref. 12). 


\begin{tabular}{|c|c|c|c|c|c|}
\hline STAGE & NUMEER OF & \multicolumn{4}{|c|}{ CHARACTER OBSERVED } \\
\hline \multirow[b]{2}{*}{ CONTAO. } & \multirow[b]{2}{*}{31} & $\begin{array}{l}\text { PRESACRAL } \\
\text { VERTEBRAE } \\
<\frac{27}{27} \frac{27}{27}\end{array}$ & $\begin{array}{l}\text { RIQS } \\
\begin{array}{l}13 . \\
<13 \text { NNT. } 14\end{array}\end{array}$ & $\begin{array}{c}\text { COSTO-STERNAL } \\
\text { JUNCTIONS } \\
\frac{7}{7} \quad \frac{7}{8} \quad \frac{8}{8}\end{array}$ & $\begin{array}{c}\text { STERNEBRAE } \\
6>6\end{array}$ \\
\hline & & & & & \\
\hline $8 \frac{1}{2}$ & 75 & & & & \\
\hline $9 \frac{1}{8}$ & 74 & & & & \\
\hline $10 \frac{1}{2}$ & 68 & & & & \\
\hline $11 \frac{1}{3}$ & 57 & & & & \\
\hline $92 \frac{1}{8}$ & 69 & & & & \\
\hline
\end{tabular}

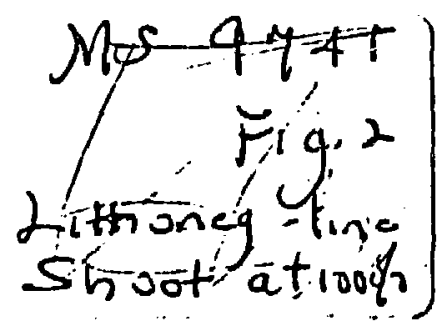


12.5 R. Experiments with $5 \mathrm{R}$ are in progress, and it appears that effects will be detectable by one of the characters, namely expression of the fourteenth rib.

III. APPLICATION OF THE SENSITIVE SYSTEMS TO THE TESTING OF CHEMICALS

Since the homeotic shifts by themselves are presumably not damaging to the organism, the endpoints are used as sensitive indicators that an agent is capable of affecting developmental processes. The quantitative relation between given degrees of

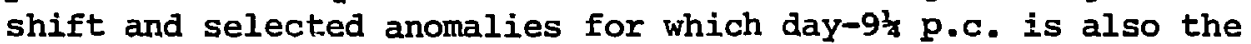
critical stage has been worked out at higher doses of $x$ rays. Is this particular quantitative relationship peculiar to the teratogen (in this case, $x$ rays), or is it a function of the existing developmental conditions? If the latter is the case, then the system can be used not merely as a sensitive detector of agents capable of causing developmental interference but as a predictor of actual levels of teratogenicity. Doses of chemical agents teratogenically equivalent to certain doses of $x$ rays can then be determined readily.

We have approached this question with one chemical, benzo(a)pyrene, BaP (Ref. 15, and Russell, unpublished). With regard to homeotic shifts (Fig. 3), an exposure to $100 \mathrm{mg} / \mathrm{kg}$ was found to be somewhat less potent than irradiation with $100 \mathrm{R} X$ rays administered at an effectively equivalent stage. (Note that the BaP was injected into the pregnant female 18 hours prior to the maximum sensitive stage, day $9 \frac{1}{4}$, in order to allow time for formation and transport of active metabolites.) Subsequent experiments have indicated that, with respect to homeotic shifts, $100 \mathrm{mg} / \mathrm{kg}$ BaP is indeed equivalent to an $x$-ray dose somewhere between 50 and $100 \mathrm{R}$; and $50 \mathrm{mg} / \mathrm{kg} \mathrm{BaP}$ is equivalent to an X-ray dose between 25 and $50 \mathrm{R}$. Significantly, this equivalence extends also to an array of axial skeleton defects induced at the corresponding stage (13), i.e., there is no clearcut qualitative difference between the actions of these two teratogens. Thus, for at least one chemical, it appears that the conditions for extrapolating from homeotic shifts to abnormalities may hold.

Both of the other low-dose systems that have been discussed, oocyte depletion and cell-cycle alteration in the telencephalon, use endpoints that in themselves are damaging to the organism. It is, therefore, perhaps not as important as in the case of the homeotic shifts to build bridges to other effects.

Some of the guidelines currently governing teratogenicity testing require preconception, postconception, and postnatal administration of the test substance; and some protocols even specify a three-generation test. Quite apart from the fact that these 
Fig.3. Homeotic shifts in four features of the axial skeleton in the $B A L B / C$ strain of mice treated on day-9lat postconception with $x$-rays, or at a developmentally equivalent stage (see text) with Benzo(a)pyrene dissolved in cornoil. Note that all distributions are shifted to the right by the treatments, with $100 \mathrm{mg} / \mathrm{kg}$ BaP being somewhat less effective than $100 \mathrm{R} \mathrm{X}$ rays. Cornoil alone has no effect. (Figure based on data by Russeil and McKinley 1978.) 


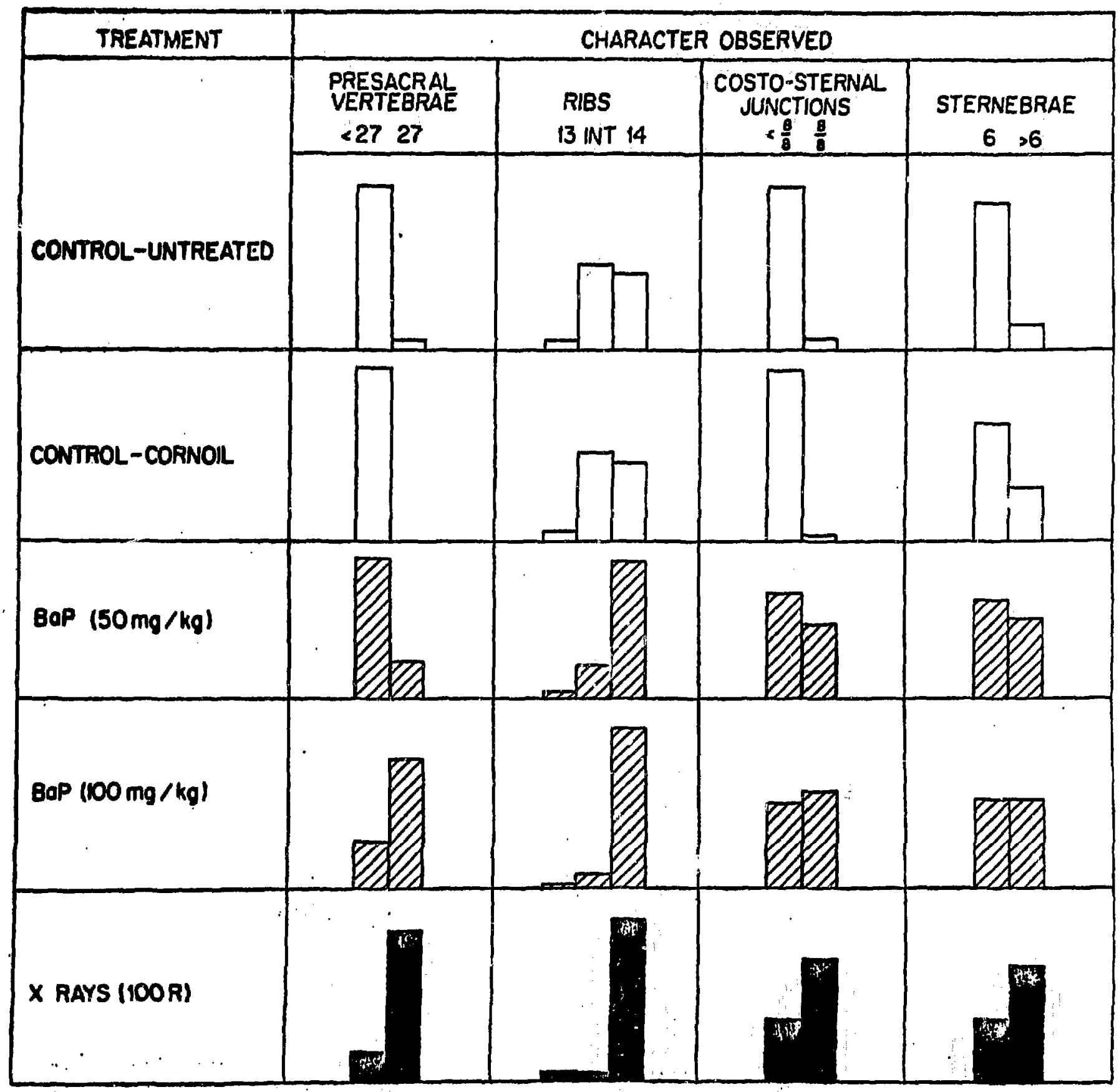


procedures confuse genetic and teratogenic effects, they also dilute the probability for discovering specific developmental damages, because only small fractions of the total dose will be received during specific critical periods. It may be suggested that tests such as the three discussed in this paper, which have been developed with strict attention to specific critical periods, can provide rapid and sensitive means for revealing whether an agent is capable of causing developmental interference. More extensive teratological investigations could then follow, if necessary. Because of metabolic differences beiween species (and even itrains) of experimental manmals, and because of the possible tissue specificity of some chemical agents, it might be well to develop a battery of indicator tests. A possible battery that would include different species, target tissues, and developmental stages, might consist of the homeotic-shift test in the mouse, oocyte killing in another species, and telencephalon changes in yet another.

\section{THE ROLE OF SENSITIVE STAGES IN ESTIMATTNG RISK}

Embryonic, fetal, and even early postnatal periods each constst of as a succession of stages that are sensitive to the induction of different arrays of effects. In experimental animals, the pattern has been determined for certain classes of endpoints but is unknown for the vast majority of possible effects. What may be surmised for man comes mainly from extrapolation to developmentally equivalent stages of the data from experimental animals.

When irvestigating effects of short-term exposures to environmental agents, epidemiological studies should pay strict attention to stage of exposure -- if possible, by week postconception. If this is not done, it is easy for a real effect to become so diluted as to be imperceptible. A specific effect inducible during only one of 38 weeks of pregnancy, for example, would virtually disappear if the sample included equal numbers derived from exposures during any cae of the 38 .

This may be illustrated by actual data for an endpoint that is apparently sensitive during several successive weeks, and therefore not quite as dilutable as if the critical period were more strictly limited. Fig. 4 depicts the microcephaly data for Hiroshima $(6,1)$ : the plot for exposures during weeks 6-11 of pregnancy shows a steep dose-response curve (albeit with wide error bars due to the smallness of the samples), while the plot for the entire period of pregnancy (with different mixes of stages at each dose po:nt) indicates a much shallower curve of different shape at the lower end. Extrapolation to low doses could presumably be different from the two tabulations. This epidemiological study was a very large one, and the sample size and dose range are not likely to be duplicated for other agents. It is thus improbable that one will be able to 
Fig.4. Incidence of microcephaly in children who had been exposed to the atomic bomb in Hiroshima while in utero. Solid line derived from children exposed weeks 6-11 postconception, broken line from all children. Error bars are standard errors of the proportions. (Eigure based on data by Miller and Mulvihill 1976; control incidence from Blot 1975.) 


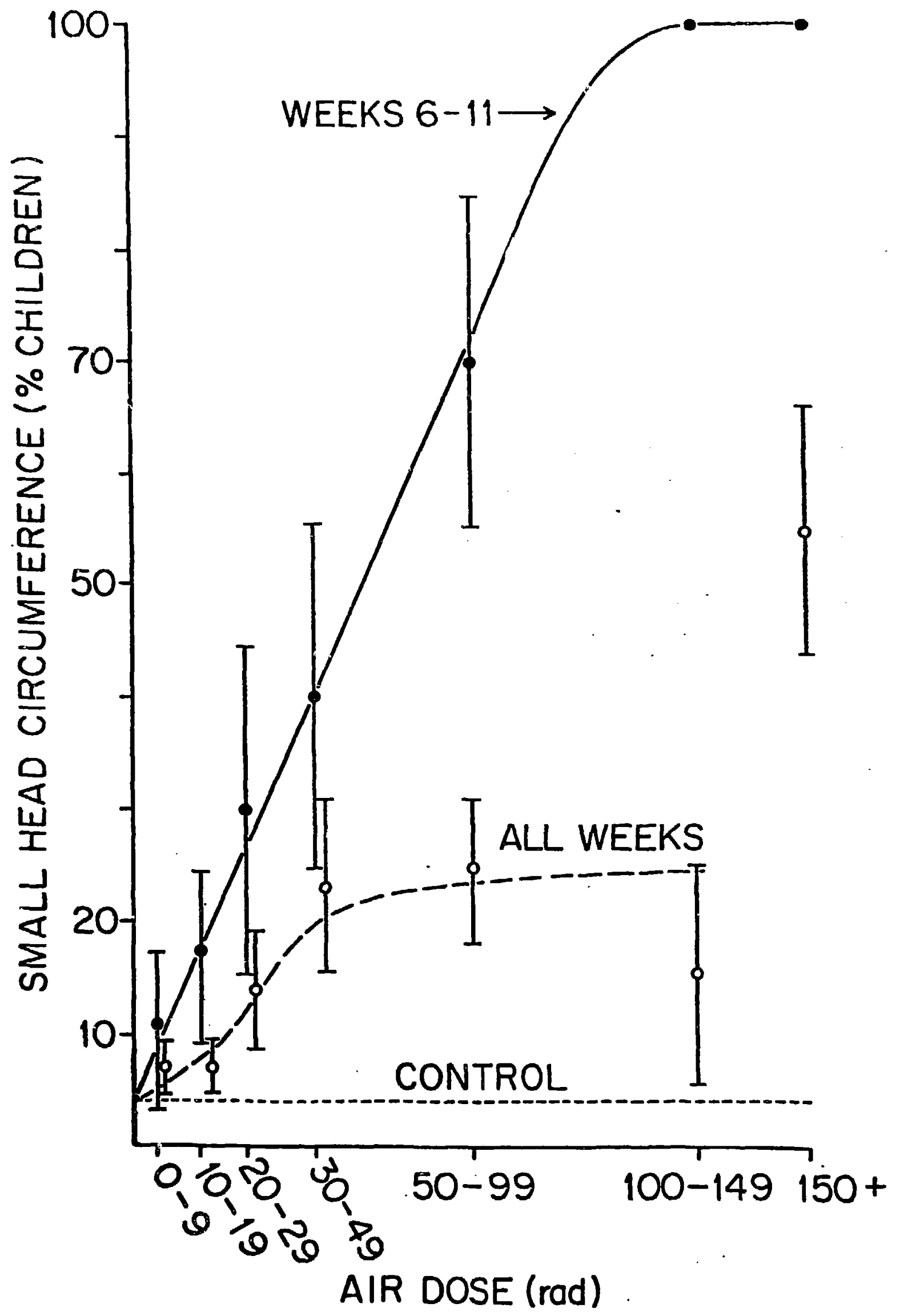


deduce, from human data alone, critical periods for other endpoints. If one cannot do so -- and therefore has to work with a smear of stages -- the low-dose extrapolation will always be an underestimate as far as the real sensitive subpopulation is concerned.

On the other hand, the circumstances that most critical periods for specific damages are probably short, may leal one to overestimate the risk from that particular damage if one assumes that protraction or fractionation of a given dose will not (or will only slightly) reduce the response. Such a spreading out of the exposure is likely to result in only a very small portion of the dose being received during the short period that is sensitive to the induction of the specified effect. For example, a single dose of $200 \mathrm{R}$ administered at one of various times during the first two weeks postconception produced a number of very severe damages; but spreading out the same dose over the entire two weeks eliminated virtually every effect. (14).

\section{SUMMARI}

Careful definition of critical periods in the development of selected characters can result in experimental systems that may be highly useful in studying risk at low levels of exposure. Three examples are presented. Epidemiological investigations can lose much of their value unless critical periods are known for the endpoints being studied. 
REFERENCES

1. Blot, W. J.: Growth and development following prenatal and childhood exposure to atomic radiation. J. Radiat. Res. Suppl., 82-88 (1975)

2. Dobson, R. I. and Kwan, T. C.: The tritium RBE at low-level exposure -- variation with dose, dose rate, and exposure duration. Current Topics in Radiation Research Quarterly $12: 44-62$ (1977)

3. Dobson, R. I., Koehler, C. G., Felton, J. S., Kwan, T. C., Wuebbles, B. J., and Jones, C. I.: Vulnerability of female germ cells in developing mice and monkeys to tritium, gamma rays, and polycyclic aromatic hydrocarbons. In Developmental Toxicology of Energy-Related Pollutants, Ed. by D. D. Mahlum et al. Techn. Info. Center, U.S. Dept. of Energy, CoNF-771017 pp. $1-14$ (1978)

4. Hicks, S. P. and D'Amato, C. J.: Low dose radiation of the developing brain. Science 14l:903-905 (1963)

5. Kameyama, Y., Hoshine, K. and Hayashi, Y.: Effects of lowdose $\mathrm{x}$-radiation on the matrix cells in the telencephalon of mouse embryos. In Developmental Toxicology of EnergyRelated Pollutants, Ed. by D. D. Mahlum et al. Techn. Info. Center, U.S. Dept. of Energy, CONF-771017, pp. 228-236 (1978)

6. Miller, R. W. and Mulvihill, J. J.: Small head size after atomic irradiation. Teratology $14: 355-358$ (1976)

7. Oakberg, E. E.: Gamma-ray sensitivity of oocytes of immature mice. Proc. Soc. Exptl. Biol. Med. 109:763-767 (1962)

8. Peters, H.: Radiation sensitivity of oocytes at different stages of development in the immature mouse. Radiat. Res. $15: 582-593$ (1961)

9. Russell, L. B.: X-ray induced developmental abnormalities in the mouse and their use in the analy-is of embryological patterns. I. External and gross visceral changes. $J$. Expt1. Zool., 114:545-602 (1950).

10. Russell, L. B.: X-ray induced developmental abrormalities in the mouse and their use in the analysis of emkryological patterns. II. Abnormalities of the vertebral column and thorax. J. Exptl. Zool., 131:329-395 (1956) 
11. Russell, L. B.: Effects of low doses of X-rays on embryonic development in the mouse. Proc. Soc. Exp. Biol. Med. 95: 174-178 (1957)

12. Russell, L. B.: Sensitivity patterns for the induction of homeotic shifts in a favorable strain of mice. Teratology $20=115-125$ (1979)

13. Russell, I. B.: Sensitivity of the homeotic-shift prescreen for environmental teratogens. Teratology 19:45A (1979)

14. Russell, L. B., Badgett, S. K., and Saylors, C. L.: Comparison of the effects of acute, continuous, and fractionated irradiation during embryonic development. Internat. J. Radiation Biol., Suppl.:343-359 (1960)

15. Russell, L. B. and McKinley, Jr., T. W.: Application of a sensitive in vivo teratological system to the testing of Benzo (a) pyrene. In Developmental Toxicology of EnergyRelated Pollutants, Ed. by D. D. Mahlum et al. Techn. Info. Center, U.S. Dept. of Energy CONF-771017, pp. 175187 (1978)

16. Fussell, L. B. and Russell, w. L.: Radiation hazards to the embryo and fetus. Radio.jogy 58:369-376 (1952)

17. Russell, L. B. and Russell, W. L.: An analysis of the changing radiation response of the developing mouse embryo. $\mathrm{J}$. Cellular Comp., Physiol., 43, Suppl. 1:103-149 (1954)

18. Russell, W. L., Russell, L. B., Steele, M. H., and Phipps, E. L. Extreme sensitivity of an immature stage of the mouse ovary to sterilization by irradiation. Science 129: 1288 (1959)

19. Wright, S.: An analysis of variability in number of digits in an inbred strain of guinea pigs. Genetics 19:506-536 (1934)

20. Wright, S.: The results of crosses between inbred strains of guinea pigs, differing in number of digits. Genetics 19: 537-551 (1934) 